\title{
XMM-Newton Observations Reveal Very High X-ray Luminosity from the Carbon-rich Wolf-Rayet Star WR 48a
}

\author{
Svetozar A. Zhekov ${ }^{1,4}$, Marc Gagné2 ${ }^{2}$ and Stephen L. Skinner ${ }^{3}$
}

\begin{abstract}
We present XMM-Newton observations of the dusty Wolf-Rayet star WR 48a. This is the first detection of this object in X-rays. The XMM-Newton EPIC spectra are heavily absorbed and the presence of numerous strong emission lines indicates a thermal origin of the WR 48a X-ray emission, with dominant temperature components at $\mathrm{kT}_{\text {cool }} \approx 1 \mathrm{keV}$ and $\mathrm{kT}_{\text {hot }} \approx 3 \mathrm{keV}$, the hotter component dominating the observed flux. No significant X-ray variability was detected on time scales $\leq 1$ day. Although the distance to WR 48a is uncertain, if it is physically associated with the open clusters Danks 1 and 2 at $\mathrm{d} \sim 4 \mathrm{kpc}$, then the resultant X-ray luminosity $\mathrm{L}_{X} \sim 10^{35} \mathrm{ergs} \mathrm{s}^{-1}$ makes it the most X-ray luminous Wolf-Rayet star in the Galaxy detected so far, after the black-hole candidate Cyg $\mathrm{X}-3$. We assume the following scenarios as the most likely explanation for the X-ray properties of WR 48a: (1) colliding stellar winds in a wide WR+O binary system, or in a hierarchical triple system with non-degenerate stellar components; (2) accretion shocks from the WR 48a wind onto a close companion (possibly a neutron star). More specific information about WR48a and its wind properties will be needed to distinguish between the above possibilities.
\end{abstract}

Subject headings: stars: individual (WR 48a) — stars: Wolf-Rayet - X-rays: stars — shock waves

\section{Introduction}

As the early surveys revealed (Pollock 1987), Wolf-Rayet (WR) stars are sources of $\mathrm{X}$-ray emission and massive $\mathrm{WR}+\mathrm{OB}$ binaries are the brightest amongst them. Pointed

\footnotetext{
${ }^{1}$ JILA, University of Colorado, Boulder, CO 80309-0440, USA; zhekovs@colorado.edu

${ }^{2}$ Department of Geology and Astronomy, West Chester University, West Chester, PA 19383; mgagne@wcupa.edu

${ }^{3}$ CASA, University of Colorado, Boulder, CO 80309-0389, USA; stephen.skinner@colorado.edu

${ }^{4}$ On leave from Space Research Institute, Sofia, Bulgaria
} 
X-ray observations with modern observatories (Chandra, XMM-Newton) confirmed this and provided us with grating spectra of WR+OB binaries (Skinner et al. 2001; Raasen et al. 2003; Schild et al. 2004; Pollock et al. 2005; Zhekov \& Park 2010b) that are rich in spectral lines from different ionic species which indicates a range of X-ray plasma temperatures. These findings suggest that the enhanced X-ray emission is produced in colliding-stellar-wind (CSW) shocks, as first proposed by Prilutskii \& Usov (1976) and Cherepashchuk (1976).

The available pointed Chandra and XMM-Newton observations suggest that the X-ray properties of presumably single WR stars might correlate with their subtype. Namely, the WN (nitrogen-rich) objects have X-ray spectra with prominent emission lines originating from an admixture of cool $(\mathrm{kT}<1 \mathrm{keV})$ and hot $(\mathrm{kT}>2 \mathrm{keV})$ plasma (Skinner et al. 2010). Only one of the four WO (oxygen-rich) stars in the Galaxy, WR 142, has been observed so far and it is a weak but extremely hard X-ray source (Oskinova et al. 2009; Sokal et al. 2010). In contrast, the single WC (carbon-rich) stars are conspicuously X-ray quiet: all the Chandra and XMM-Newton pointed observations of WCs so far have resulted in non-detections (Oskinova et al. 2003; Skinner et al. 2006; Skinner et al. 2009). The X-ray production mechanism in single WR stars is still not well-understood, but the ubiquitous presence of a high-temperature X-ray component in WR spectra is a clear indication that other processes besides embedded, radiative wind shocks are contributing (Skinner et al. 2010 and references therein).

Overall, binary systems, especially wide binaries, are more X-ray luminous than comparable single WR stars. For single WR stars $L_{X}$ is typically in the range $10^{31}-10^{33}$ ergs $\mathrm{s}^{-1}$ (Skinner et al. 2010), while for the known wide WR+OB binaries it is about an order of magnitude higher, with WR 140 having the largest known luminosity of $(1-2) \times 10^{34}$ ergs $\mathrm{s}^{-1}$ (Pollock et al. 2005). As a rule, if a WR star is detected with $L_{\mathrm{X}}>10^{33} \mathrm{ergs} \mathrm{s}^{-1}$, it is most likely a wide CSW binary system.

In this Letter, we report the first X-ray detection of the Wolf-Rayet star WR 48a, which establishes it as a very luminous X-ray source and as such, a likely multiple system.

\section{The Wolf-Rayet Star WR 48a}

WR 48a was discovered in a near-infrared survey and originally classified as a WC9 object (Danks et al. 1983) with a current spectral classification of WC8 (van der Hucht 2001). WR 48a is located inside the G305 star-forming region in the Scutum Crux arm of the Galaxy. Within $2^{\prime}$ of WR 48a are two compact infrared clusters (Danks 1 and 2) and its proximity to them suggests that this WR star likely originates from one or the other (Danks et al. 1984). 
The optical extinction towards WR 48a is very high, $\mathrm{A}_{V}=9.2 \mathrm{mag}$ (Danks et al. 1983), with a small fraction of it ( $\sim 2 \mathrm{mag}$ ) coming from circumstellar material (Baume et al. 2009).

The infrared variability of WR 48a suggests that it is a long-period episodic dustmaker (Williams 1995), but the dust-formation history indicates that the actual situation might be more complex. Namely, Williams et al. (2003) proposed the existence of a shortperiod $(P \approx 1 \mathrm{yr})$ binary in addition to the longer-period binary $(P>23 \mathrm{yr})$, where the latter is thought to cause the episodic dust formation. High-angular resolution near-infrared observations revealed a 70 mas size for the dust shell in WR 48a (Monier et al. 2007).

The distance to WR 48a is not well constrained. If it is physically associated with the open clusters Danks 1 and 2, Danks et al. (1983) estimated a distance of $4 \mathrm{kpc}$, but van der Hucht (2001) adopted a photometric distance of $1.21 \mathrm{kpc}$ based on a WC8 spectral classification.

No information is available for the mass-loss rate and velocity of the stellar wind in WR 48a. But, indirect evidence of its powerful wind comes from mid-infrared observations of the region surrouding WR 48a (Clark \& Porter 2004), which reveal a three-lobed nebula whose morphology is suggestive of a wind-blown structure and triggered massive star formation.

\section{Observations and Data Reduction}

WR 48a was observed with XMM-Newton on Jan 9, 2008. As discussed below, the presence of numerous spectral lines and our spectral analysis show that the X-ray emission is thermal and is heavily absorbed. The high absorption masks most emission in the RGS energy range below $2 \mathrm{keV}$, so we were not able to obtain any valuable information from the grating spectra. Thus, this study is based on the XMM-Newton European Photon Imaging Camera (EPIC) data which after excluding background flares resulted in effective exposures of $57 \mathrm{ksec}$ for the pn spectrum and $71 \mathrm{ksec}$ per MOS spectrum. The corresponding total number of X-ray counts in the $(0.5-10 \mathrm{keV})$ energy range was: 75,000 (pn), 51,000 (MOS1) and 50,000 (MOS2). We used the XMM-Newton Science Analysis System (version 10.0.0) to extract the source and background spectra and to construct the corresponding response matrices and ancillary response files. We used standard as well as custom models in version 11.3.2 of XSPEC (Arnaud 1996) for our spectral analysis.

Figure 1 shows the soft and hard-band images of WR 48a that illustrate an excellent correspondence between the X-ray source and the optical coordinates of this star (J2000): $\alpha_{X M M}=13^{h} 12^{m} 39.47, \delta_{X M M}=-62^{\circ} 42^{\prime} 56^{\prime \prime} .00 ; \alpha_{S I M B A D}=13^{h} 12^{m} 39.65, \delta_{S I M B A D}=$ 
$-62^{\circ} 42^{\prime} 55^{\prime \prime} 80$. There is an optical star located $\sim 9^{\prime \prime}$ from WR 48a in the southwest direction (Wallace et al. 2003) but we find no detectable X-ray emission from that source. Thus, all the EPIC X-ray emission should be associated with WR 48a1.

\section{Global Spectral Fits}

A general property of the XMM-Newton spectrum of WR 48a is that it is heavily absorbed and most of the X-ray photons are at energies $>1 \mathrm{keV}$. We thus re-binned the pn, MOS1, and MOS2 EPIC spectra in the 0.5 - $10 \mathrm{keV}$ range to a minimum of 100 counts per bin and fitted them simultaneously. We used discrete-temperature plasma models to derive the global properties of the X-ray emitting plasma in WR 48a. We considered two limiting cases: (i) models that assume plasma in collisional ionization equilibrium (CIE), and (ii) plasma with non-equilibrium ionization (NEI). For consistency in the atomic data, we made use of emission models vapec and vpshock in XSPEC. Since no specific information is available on the WR 48a abundances, we adopted the following set of abundances typical for the WC stars (by number): $\mathrm{H}=0.00, \mathrm{He}=0.618, \mathrm{C}=0.248, \mathrm{~N}=0.00, \mathrm{O}=0.120$, $\mathrm{Ne}=1.15 \times 10^{-2}, \mathrm{Mg}=1.68 \times 10^{-3}, \mathrm{Si}=4.23 \times 10^{-4}, \mathrm{~S}=9.40 \times 10^{-5}, \mathrm{Fe}=2.36 \times 10^{-4}$ (van der Hucht et al. 1986). Ar and Ca are not present in the van der Hucht et al. (1986) abundance set, so assumed a fiducial value of $1.2 \times 10^{-5}$. We varied the $\mathrm{Ne}, \mathrm{Mg}, \mathrm{Si}, \mathrm{S}, \mathrm{Ar}$, $\mathrm{Ca}$ and $\mathrm{Fe}$ abundances in the spectral fits since these elements provide most of the X-rays at energies $\geq 1 \mathrm{keV}$. Table 1 and Figure 2 present the results from the two-temperature model spectral fits of WR 48a.

Though the quality of the two-temperature vapec model fit is acceptable, the NEI vpshock model provides a better fit to the data. Applying the Gorenstein (1975) conversion $\left(\mathrm{N}_{H}=2.22 \times 10^{21} \mathrm{~A}_{V} \mathrm{~cm}^{-2}\right)$, we find that the X-ray absorption is consistent (within 10-15\%) with the optical extinction $\left(\mathrm{A}_{V}=9.2 \mathrm{mag} ; \S 2\right.$ 2). But, the X-ray data may indicate some extra absorption $(\sim 40-50 \%)$ if a more recent conversion is used, based on studies of starforming regions: $\mathrm{N}_{H}=(1.6-1.7) \times 10^{21} \mathrm{~A}_{V} \mathrm{~cm}^{-2}$ (Vuong et al. 2003; Getman et al. 2005). The derived abundance values are in general consistent with the adopted set of non-solar WC abundances. However, the abundance values derived from X-ray analysis and especially from CCD spectra should be considered with caution. Also, due to the high X-ray absorption no valuable information could be derived for the abundances of carbon, nitrogen and oxygen.

\footnotetext{
${ }^{1} \mathrm{WR} 48 \mathrm{a}$ is also detected with Chandra (ObsId: 8922) but pileup in the ACIS CCD is so high that no reliable spectral or timing information for WR 48a could be extracted. The ACIS image confirms that the WR 48a identification is correct (J2000: $\left.\alpha_{\text {Chandra }}=13^{h} 12^{m} 39.63, \delta_{\text {Chandra }}=-62^{\circ} 42^{\prime} 55^{\prime \prime} .90\right)$ and no other sources contribute to its X-ray emission (Gagné et al. 2010, in preparation).
} 
But perhaps the most important result from the global fits is that at least two components with different plasma temperatures are needed to obtain an acceptable representation of the observed spectrum, and the hot component requires a relatively high temperature. The hot component $(\mathrm{kT} \approx 2.8-3.2 \mathrm{keV})$ provides about $75-85 \%$ of the observed flux, while the cooler component $(\mathrm{kT} \approx 0.9-1.1 \mathrm{keV})$ provides $\sim 60 \%$ of the unabsorbed X-ray emission from this WC star.

In addition to the two-component models, we ran some more complex models: (i) a model with a thermal CIE plasma distribution based on our custom model which is similar to c6pvmkl in XSPEC, but uses the apec collisional plasma for the X-ray spectrum at a given temperature; and (ii) a model with a distribution of adiabatic NEI shocks which made use of our custom XSPEC model which was successfully used in the analysis of the X-ray spectra of SNR 1987A (e.g., Zhekov et al. 2009, and the references therein). Due to the high X-ray absorption, the X-ray spectral fits of WR 48a are not sensitive to the presence of very cool plasma. Thus, we considered only plasma temperatures above $0.5 \mathrm{keV}$ for the emission measure distribution in WR 48a. The fit results are given in Table 1, Figure 3 shows that the derived distribution of emission measure is bimodal, with temperature peaks closely matching the components of the two-temperature models.

From the above results, it seems reasonable to conclude that there is evidence for a two-temperature distribution in the X-ray emitting region of WR 48a. But, it is worthwhile to treat such results with caution when based on CCD spectra since the spectral lines are not well resolved and the lines (and their ratios) are most sensitive to the presence of a variety of plasma temperatures. But, we believe that the bimodal distribution is in fact an indicator of a temperature-stratified X-ray emission region in WR 48a.

\section{Discussion}

The two most important results from the model fits to the XMM-Newton spectra of WR 48a are that it is a very luminous X-ray source and thermal plasmas with high temperature dominate its emission (Table 1 and Fig. 3). Using the unabsorbed flux values from Table 1, the X-ray luminosity of WR 48a is $\mathrm{L}_{X}(0.5-10 \mathrm{keV})=(0.5-2.1) \times 10^{34} d_{k p c}^{2}$ ergs $\mathrm{s}^{-1}$, where $d_{k p c}$ is the distance in units of kpc. The upper limit is from the NEI shock model which gives a better quality of fit. The distance to WR 48a is not tightly constrained and a range of values is available in the literature: $d_{k p c}=1.21-4(\S 2)$. But, based on its proximity to the open clusters Danks 1 and 2 and the similar interstellar extinction (e.g., Danks et al. 1983; Clark \& Porter 2004; Baume et al. 2009), the distance of $4 \mathrm{kpc}$ seems more realistic, which puts the X-ray luminosity in the range $\mathrm{L}_{X}=(0.8-3.4) \times 10^{35} \mathrm{ergs} \mathrm{s}^{-1}$. In this case 
and excluding the most X-ray luminous WR star, Cyg X-3, which is a WR binary with a compact companion (a neutron star or a black hole), WR 48a is the most luminous WR star in the Galaxy among those observed so far. For example, the X-ray luminosity of the brightest CSW binary, WR 140, is: $(0.5-3) \times 10^{34} \mathrm{ergs} \mathrm{s}^{-1}$ (Zhekov \& Skinner 2000; Pollock et al. 2005). Adopting the bolometric luminosity from Clark \& Porter (2004), we note the very high value of $\lg L_{X} / L_{b o l}=[-4.3,-3.7]$ for WR 48a. All this raises the interesting question about the X-ray production mechanism in WR 48a.

Colliding Stellar Winds. As already mentioned, WR 48a is an episodic dust-maker (Williams 1995) which suggests that CSWs in a wide binary system with an eccentric orbit might provide most of its X-ray emission, as is the case for the prototype episodic dust-maker amongst WR stars, WR 140 (Williams et al. 1990). Unfortunately, the lack of information about the stellar wind parameters of WR 48a did not allow us to make a comparison between the theoretical predictions based on hydrodynamic CSW modeling and observations. On the other hand, some simple (e.g. qualitative) considerations are possible. The temperature of the hot plasma component deduced from the global spectral fits (\$4) can provide an estimate of the stellar wind velocity (see $\$ 5.2$ in Zhekov 2007 for discussion of CSW models versus discrete-temperature models ). For the case of typical WC abundances, the postshock plasma temperature is $\mathrm{kT}=3.09 V_{1000}^{2} \mathrm{keV}$, where $V_{1000}$ is the shock velocity in units of $1000 \mathrm{~km}$ $\mathrm{s}^{-1}$. From Table 1 and Fig. 3, we see that the stellar wind of the WC star in WR 48a must have a velocity $\mathrm{V}_{\text {wind }} \geq 1000 \mathrm{~km} \mathrm{~s}^{-1}$. Such high velocities would be expected for WC8-9 stars since they have typical average wind speeds $1400 \mathrm{~km} \mathrm{~s}^{-1}$ (Prinja et al. 1990), $1300 \mathrm{~km}$ $\mathrm{s}^{-1}$ (Eenens \& Williams 1994). We note that the derived values for ionization age of the shocks in the two-temperature model (Table 1) and in the model with a distribution of NEI shocks (Fig. 3) are also qualitatively consistent with the CSW picture. Namely, the higher density and higher temperature plasma is located near the axis of symmetry (the line-ofcenters between the two stars) while the less dense and cooler plasma is found downstream from that axis. Thus, it is natural in the CSW picture that the higher temperature plasma will have a larger ionization age. We can use the scaling law for the CSW X-ray luminosity with the mass-loss rate $(\dot{M})$, wind velocity $(v)$ and binary separation $(D): L_{X} \propto \dot{M}^{2} v^{-3} D^{-1}$ (Luo et al. 1990; Mvasnikov \& Zhekov 1993) for a comparison between WR 48a and the classical CSW binary WR 140. Adopting the WR 140 binary parameters (Williams et al. 1990; Pollock et al. 2005), a wind velocity of $1300-1400 \mathrm{~km} \mathrm{~s}^{-1}$ in WR 48a and assuming that its mass loss is equal to that of WR 140, we see that the binary separation in WR 48a could be similar to that in WR 140 even when the WR 48a X-ray luminosity is about an order of magnitude larger than that of WR 140 (see above). Therefore, the CSW picture in a wide binary system is at least qualitatively consistent with the observational data for WR 48a. 
Magnetically Confined Wind Shocks (MCWS). This mechanism is capable of producing hard X-ray emission. It was proposed to explain the high plasma temperature found in X-ray emission from young massive stars and requires the presence of a relatively strong magnetic field (Schulz et al. 2003, Gagné et al. 2005 and references therein). Then, could it be that the WC star in WR 48a is a magnetized object or has a close magnetized companion? We note that the X-ray luminosity of the prototype MCWS object, $\theta^{1}$ Ori $\mathrm{C}$, is $\mathrm{L}_{X}(0.5-10 \mathrm{keV}) \approx$ $10^{33}$ ergs s$^{-1}$ (Gagné et al. 2005) which is one to two orders of magnitude below that of WR 48a. Thus, we can likely rule out the case of a close magnetized companion. On the other hand, the MCWS mechanism does not seem promising if adopted directly to the WC star in WR 48a. First, in order to be efficient this mechanism requires a relatively strong global magnetic field to confine the massive WR wind and no such fields have been reported at present for these stars. Second, because of the expected decay of the magnetic field strength with the age of a massive star, the MCWS mechanism is associated only with young massive stars (age $\leq 1$ Myr; Schulz et al. 2003). Thus, the likely association of WR 48a with the open clusters Danks 1, 2 and the recent estimate of $\sim 5$ Myr for their age (Baume et al. 2009) present serious difficulties for the MCWS model in the case of WR 48a.

Wind Accretion Shocks. The presence of a close degenerate companion (e.g. a neutron star) seems very intriguing and accretion onto such an object can in general provide high X-ray luminosities (e.g., Davidson \& Ostriker 1973). Details depend on the actual binary parameters and wind properties of the main stellar component which are yet unknown for WR 48a. But, this case may not be unlikely if WR 48a is indeed a member of the 5-Myrold open clusters Danks 1, 2 and if it once had a more massive close companion which has already evolved and exploded as a supernova.

We have listed above some physical mechanisms that might be able to explain the observed X-ray properties of WR 48a. One could continue this list by considering other mechanisms or even more complex combinations of those mentioned above. But, all such mechanisms and the corresponding physical picture remain quite speculative because of the scarcity of detailed observational data for WR 48a. Thus, we will end our discussion by simply mentioning the cases that look least speculative to us and briefly discuss what observational information may help distinguish between them.

For the moment, the following cases are the most likely explanation for the X-ray properties of WR 48a: (i) CSWs in a wide binary system; or (ii) accretion wind shocks in a close binary: WR+compact companion (a neutron star). Variability is a key parameter for distinguishing between these cases. Namely, if long-term (months, years) X-ray variability is established from future observations, then case (i) would be strongly favored. Furthermore, case (i) would be strengthened if variable non-thermal radio (NTR) emission from 
WR 48a is eventually discovered and even more so if the X-ray emission is found to modulate at a similar period. In general, NTR WRs are commonly associated with wide binary systems (Dougherty \& Williams 2000). Alternatively, if short-term (a few days) variability is established or a sudden change of the X-ray luminosity is detected, case (ii) would be favored. The $X M M-N e w t o n$ data show no significant variability on timescales $\leq 1$ day; the Kolmogorov-Smirnov test rules out variability at the $95 \%$ confidence level. On the other hand, based on the infrared variability of WR 48a, Williams et al. (2003) proposed that it is a triple stellar system. If so, we may expect some very long-period variability due to the X-rays from CSWs in a wide binary with eccentric orbit and some with a much shorter period that could result from the stellar wind shocking onto a non-degenerate companion. In this case, WR 48a would be very similar to the CSW binary WR 147 which was recently resolved into a double X-ray source with Chandra, of which one component is variable and possibly an unresolved binary (Zhekov \& Park 2010a,b). However, WR 48a has a much higher X-ray luminosity. Finally, we hope that the unusually high $\mathrm{L}_{X}$ of WR 48a will motivate deeper follow-up observations across the entire spectrum (radio, infrared, optical, UV, X-rays). Such observations would provide us with valuable information about the star and its wind properties that could be very helpful for constraining the physical picture in this remarkable but understudied Wolf-Rayet system.

This work was supported by NASA through Chandra Award GO8-9014X and NASA Award NNX08AO69G to the West Chester University, West Chester, Pennsylvania. SAZ

acknowledges financial support from Bulgarian National Science Fund grant DO-02-85. The authors thank an annonymous referee for his/her comments and suggestions.

Facilities: XMM-Newton (EPIC).

\section{REFERENCES}

Arnaud, K.A. 1996, in Jacoby G., Barnes, J. eds., ASP Conf. Ser. Vol. 101, Astronomical Data Analysis Software and Systems, Astron. Soc. Pac., San Francisco, 17

Baume, G., Carraro, G., \& Momany, Y. 2009, MNRAS, 398, 221

Cherepashchuk, A.M. 1976, Soviet Astronomy Letters, 2, 138

Clark, J.S., \& Porter, J.M. 2004, A\&A, 427, 839 
Danks, A.C., Dennefeld, M., Wamsteker, W, \& Shaver, P.A. 1983, A\&A, 118, 301

Danks, A.C., Wamsteker, W, Shaver, P.A. \& Retallack, D.S. 1984, A\&A, 132, 301

Davidson, K., \& Ostriker, J.P. 1973, ApJ, 179, 585

Dougherty, S.M., \& Williams, P.M. 2000, MNRAS, 319, 1005

Eenens, P.R.J, \& Williams, P.M. 1994, MNRAS, 260, 1082

Gagné, M., Oksala, M.E., Cohen, D.H., Tonnesen, S.K., ud-Doula, A., Owocki, S.P. Townsend, R.H.D., \& MacFarlane, J.J. 2005, ApJ, 628, 986

Getman, K.V., Feigelson, E.D., Grosso, N., McCaughrean, M.J., Micela, G., Broos, P., Garmire, G., \& Townsley, L. 2005, ApJS, 160, 363

Gorenstein, P. 1975, ApJ, 198, 95

Luo, D., McCray, R., \& MacLow, M.-M. 1990, ApJ, 362, 267

Monier, J.D., Tuthill, P.G., Danchi, W.C., Murphy, N., \& Harries, T.J. 2007, ApJ, 655, 1033

Myasnikov, A.V. \& Zhekov, S.A. 1993, MNRAS, 260, 221

Oskinova, L.M, Ignace, R., Hamann, W.-R., Pollock, A.M.T., \& Brown, J.C. 2003, A\&A, 402,755

Oskinova, L.M, Hamann, W.-R., Feldmeier, A., Ignace, R., Chu, Y.-H. 2009, ApJ, 693, L44

Pollock, A.M.T. 1987, ApJ, 320, 283

Pollock, A.M.T., Corcoran, M.F., Stevens, I.R., \& Williams, P.M. 2005, ApJ, 629, 482

Prilutskii, O.F \& Usov, V.V. 1976, Soviet Astronomy, 20, 2

Prinja, R.K., Barlow, M.J., \& Howarth, I.D. 1990, ApJ, 361, 607

Raassen, A.J.J., van der Hucht, K.A., Mewe, R., Antokhin, I.I., Rauw, G., Vreux, J.-M., Schmutz, W., \& Güdel, M. 2003, A\&A, 402, 653

Schild, H. et al. 2004, A\&A, 422, 177

Schulz, N.S., Canizares, C., Huenemoerder, D., \& Tibbets, K. 2003, ApJ, 595, 365

Skinner, S.L., Güdel, M., Schmutz, W. \& Stevens, I.R. 2001, ApJ, 558, L113 
Skinner, S.L., Güdel, M., Schmutz, W.\& Zhekov, S.A. 2006, Ap\&SS, 304, 97

Skinner, S.L., Zhekov, S.A., Güdel, M., Schmutz, W. \& Sokal, K.R. 2009, BAAS, 41, 470

Skinner, S.L., Zhekov, S.A., Güdel, M., Schmutz, W. \& Sokal, K.R. 2010, AJ, 139, 825

Sokal, K.R., Skinner, S.L., Zhekov, S.A., Güdel M., \& Schmutz W. 2010, ApJ, 715, 1327

van der Hucht, K.A. 2001, New Astronomy Rev., 45, 135

van der Hucht, K.A., Cassinelli, J.P., \& Williams P.M. 1986, A\&A, 168, 111

Vuong, M.H., Montmerle, T., Grosso, N., Geigelson, E.D., Verstraete, L., \& Ozawa, H. 2005, A\&A, 408, 581

Wallace, D.J., Moffat, A.F.J., Shara, M.M., Gies, D.R., Niemela, V.S., \& Nelan, E. 2003, 'A Massive Star Odyssey, from Main Sequence to Supernova', Proceedings, IAU Symposium no. 212, eds. K.A. van der Hucht, A. Herrero \& C. Esteban, Astronomical Society of the Pacific, San Francisco, 578

Williams, P.M. 1995, 'Wolf-Rayet Stars: binaries, colliding winds, evolution', Proceedings IAU Symposium no. 163, eds. K.A. van der Hucht and P.M.Williams, Kluwer Academic Publishers, Dordrecht, 335

Williams, P.M., van der Hucht, K.A., Morris, P.W., \& Marang, F. 2003, 'A Massive Star Odyssey, from Main Sequence to Supernova', Proceedings, IAU Symposium no. 212, eds. K.A. van der Hucht, A. Herrero \& C. Esteban, Astronomical Society of the Pacific, San Francisco, 115

Williams, P.M., van der Hucht, K.A., Pollock, A.M.T., Florkowski, D.R., van der Woerd, H., \& Wamsteker, W. 1990, MNRAS, 243, 662

Zhekov, S.A. 2007, MNRAS, 382, 886

Zhekov, S.A., McCray , R., Dewey, D., Canizares, C.R., Borkowski, K.J., Burrows, D.N. \& Park, S. 2009, ApJ, 692, 1190

Zhekov, S.A., \& Park, S. 2010a, ApJ, 709, L119

Zhekov, S.A., \& Park, S. 2010b, ApJ, 721, 518

Zhekov, S.A. \& Skinner, S.L. 2000, ApJ, 538, 808 

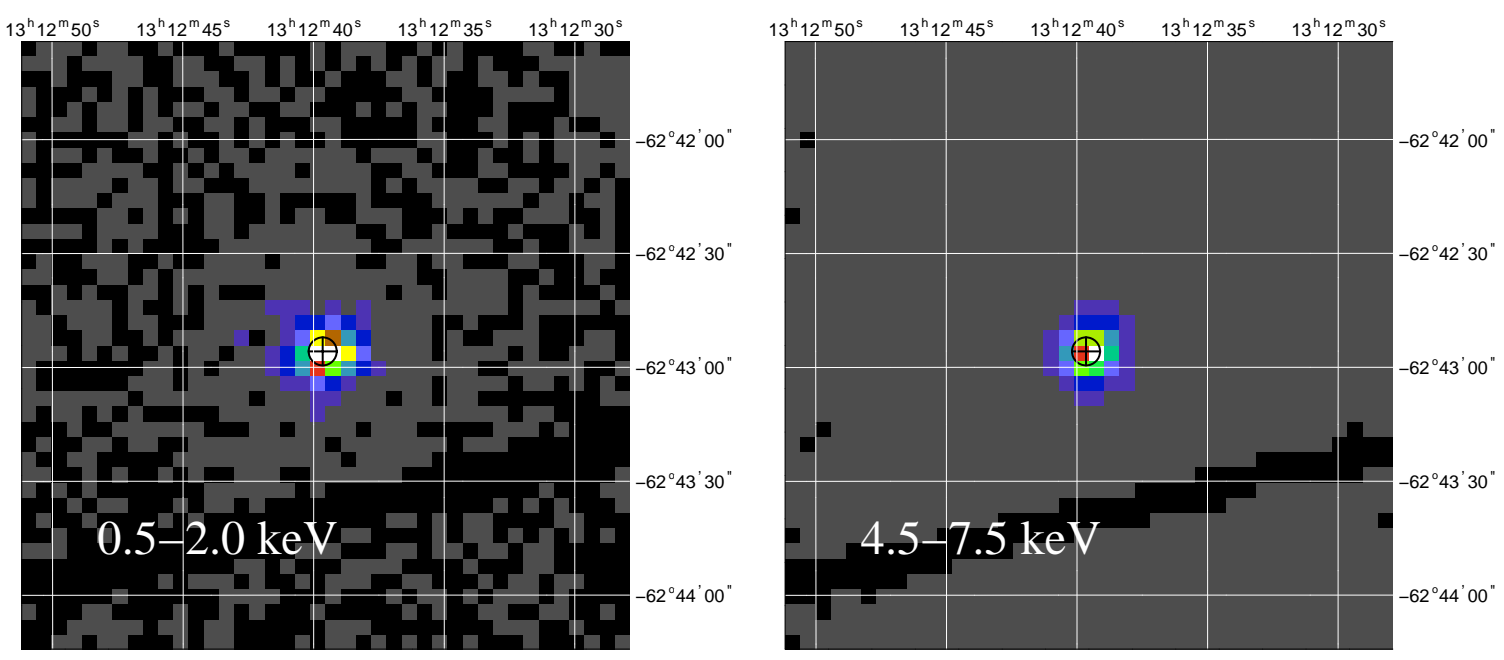

Fig. 1.- The EPIC-pn images of WR 48a (linear intensity scale). R.A. (J2000) and decl. (J2000) are on the horizontal and vertical axes, respectively. The circled plus sign gives the optical position of WR 48a (SIMBAD). 

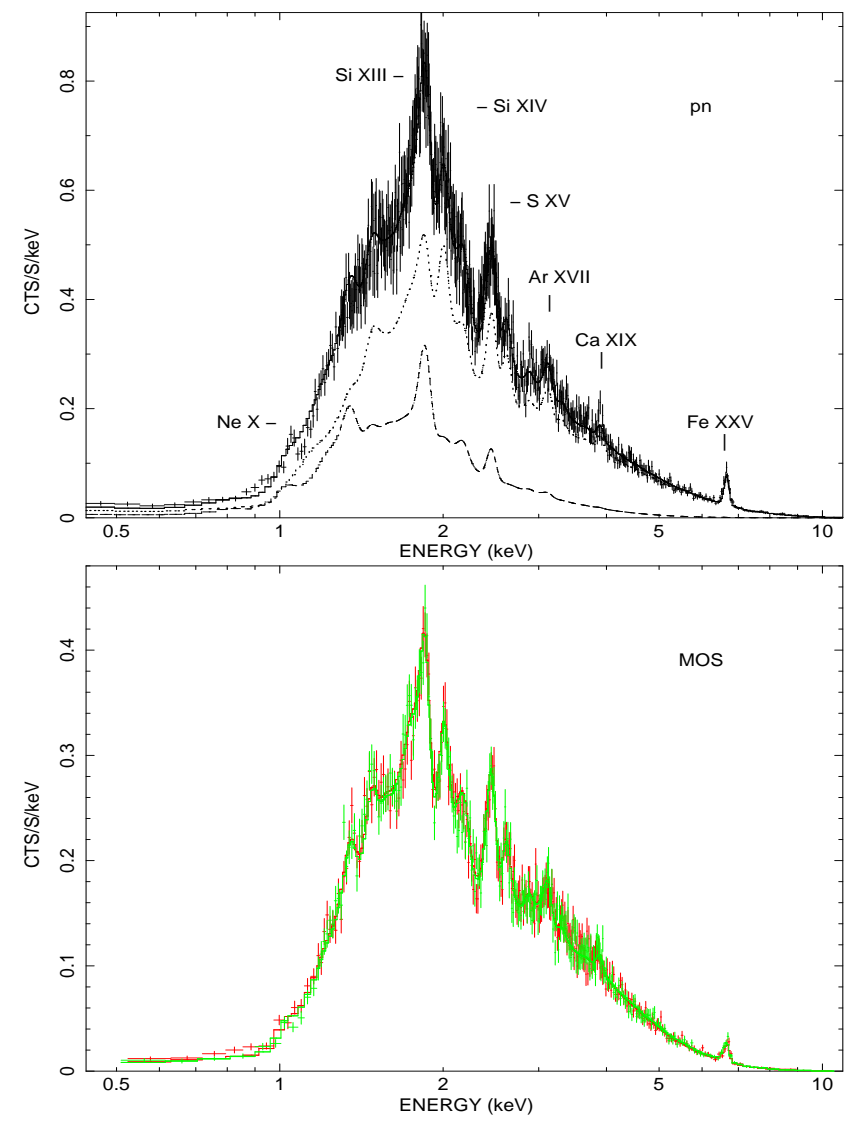

Fig. 2.- XMM-Newton background-subtracted spectra of WR 48a and the two-temperature fit with NEI shocks (Table 1). The MOS1 and MOS2 spectra are drawn in red and green, respectively. The low and high-temperature components are shown in the upper panel with dashed and dotted lines, respectively. 


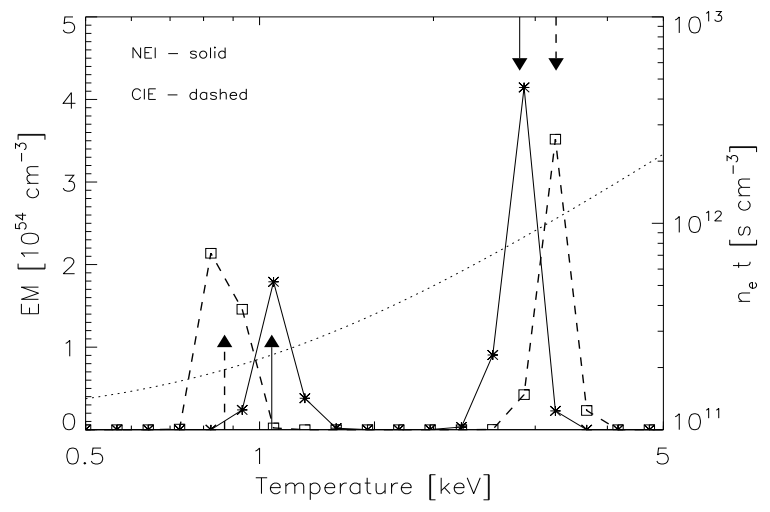

Fig. 3.- Emission measure $\left(\mathrm{EM}=\int n_{e} n_{\mathrm{He}} d V\right)$ of the WR 48a distribution of thermal CEI plasma and of adiabatic NEI shocks for a reference distance of $d=1 \mathrm{kpc}$. The arrows indicate the plasma temperature from the two-temperature fits (see Table 1). The dotted line shows the derived ionization age in the case of NEI shocks $\left(n_{e} t\right)$. 
Table 1. Global Spectral Model Results

\begin{tabular}{lllll}
\hline \hline \multicolumn{1}{c}{ Parameter } & 2T vapec & 2T vpshock & CIE Plasma & NEI Shocks \\
\hline$\chi^{2} / \mathrm{dof}$ & $1382 / 1066$ & $1169 / 1064$ & $1403 / 1064$ & $1173 / 1062$ \\
$\mathrm{~N}_{H}\left(10^{22} \mathrm{~cm}^{-2}\right)$ & $2.27_{-0.02}^{+0.03}$ & $2.30_{-0.04}^{+0.05}$ & $2.29_{-0.03}^{+0.03}$ & $2.29_{-0.04}^{+0.04}$ \\
$\mathrm{kT}_{1}(\mathrm{keV})$ & $0.87_{-0.01}^{+0.01}$ & $1.05_{-0.07}^{+0.09}$ & & \\
$\mathrm{kT}_{2}(\mathrm{keV})$ & $3.26_{-0.07}^{+0.06}$ & $2.82_{-0.07}^{+0.07}$ & & \\
$\mathrm{EM}_{1}\left(10^{54} \mathrm{~cm}^{-3}\right)$ & $3.58_{-0.07}^{+0.10}$ & $2.42_{-0.16}^{+0.08}$ & & \\
$\mathrm{EM}_{2}\left(10^{54} \mathrm{~cm}^{-3}\right)$ & $4.21_{-0.04}^{+0.06}$ & $5.34_{-0.04}^{+0.04}$ & & \\
$\tau_{1}\left(10^{11} \mathrm{~cm}^{-3} \mathrm{~s}\right)$ & & $2.42_{-0.42}^{+0.56}$ & & \\
$\tau_{2}\left(10^{11} \mathrm{~cm}^{-3} \mathrm{~s}\right)$ & & $8.09_{-1.02}^{+1.39}$ & & \\
$\mathrm{Ne}$ & $0.46_{-0.09}^{+0.10}$ & $0.11_{-0.01}^{+0.03}$ & $0.53_{-0.12}^{+0.16}$ & $0.11_{-0.03}^{+0.03}$ \\
$\mathrm{Mg}$ & $0.23_{-0.04}^{+0.03}$ & $0.13_{-0.02}^{+0.02}$ & $0.25_{-0.04}^{+0.04}$ & $0.13_{-0.02}^{+0.02}$ \\
$\mathrm{Si}$ & $1.35_{-0.06}^{+0.06}$ & $0.64_{-0.05}^{+0.06}$ & $1.38_{-0.07}^{+0.07}$ & $0.65_{-0.06}^{+0.06}$ \\
$\mathrm{~S}$ & $4.90_{-0.20}^{+0.23}$ & $1.78_{-0.12}^{+0.15}$ & $4.88_{-0.20}^{+0.20}$ & $1.81_{-0.12}^{+0.12}$ \\
$\mathrm{Ar}$ & $9.72_{-0.92}^{+0.94}$ & $2.78_{-0.39}^{+0.19}$ & $9.58_{-0.94}^{+1.03}$ & $2.82_{-0.34}^{+0.38}$ \\
$\mathrm{Ca}$ & $5.19_{-0.62}^{+0.65}$ & $1.97_{-0.33}^{+0.34}$ & $5.22_{-0.70}^{+0.69}$ & $2.00_{-0.41}^{+0.33}$ \\
$\mathrm{Fe}$ & $1.36_{-0.07}^{+0.06}$ & $1.31_{-0.07}^{+0.07}$ & $1.37_{-0.07}^{+0.07}$ & $1.30_{-0.07}^{+0.07}$ \\
$\mathrm{~F}_{X}\left(10^{-11} \mathrm{ergs} \mathrm{cm}^{-2} \mathrm{~s}^{-1}\right)$ & $1.06(4.21)$ & $1.05(17.7)$ & $1.06(4.30)$ & $1.05(17.8)$ \\
$\mathrm{F}_{X, h o t}\left(10^{-11} \mathrm{ergs} \mathrm{cm}^{-2} \mathrm{~s}^{-1}\right)$ & $0.82(1.79)$ & $0.89(6.98)$ & $0.81(1.79)$ & $0.89(6.81)$ \\
\hline
\end{tabular}

Note. - Results from simultaneous fits to the EPIC spectra of WR 48a. Tabulated quantities are the neutral hydrogen absorption column density $\left(\mathrm{N}_{H}\right)$, plasma temperature $(\mathrm{kT})$, emission measure $\left(\mathrm{EM}=\int n_{e} n_{H e} d V\right.$; see also Fig. 3) for a reference distance of $\mathrm{d}=1 \mathrm{kpc}$, shock ionization age $\left(\tau=n_{e} t\right)$, the absorbed X-ray flux $\left(\mathrm{F}_{X}\right)$ in the $0.5-10$ $\mathrm{keV}$ range followed in parentheses by the unabsorbed value $\left(\mathrm{F}_{X, h o t}\right.$ denotes the highertemperature component, $\mathrm{kT}_{2}$; or $\mathrm{kT} \geq 2 \mathrm{keV}$ for CIE Plasma and NEI Shocks). The derived abundances are with respect to the typical WC abundances (van der Hucht et al. 1986). Errors are the $1 \sigma$ values from the fits. 